Supplement of Earth Syst. Dynam., 12, 837-855, 2021

https://doi.org/10.5194/esd-12-837-2021-supplement

(C) Author(s) 2021. CC BY 4.0 License.

(c) (1)

Supplement of

\title{
Multiscale fractal dimension analysis of a reduced order model of coupled ocean-atmosphere dynamics
}

Tommaso Alberti et al.

Correspondence to: Tommaso Alberti (tommaso.alberti@inaf.it)

The copyright of individual parts of the supplement might differ from the article licence. 


\section{Supplement}

\section{S1 A practical guide to evaluate the generalized fractal dimensions}

A fractal dimension is a characteristic measure of scale invariance describing how the details of an object (e.g., a dynamical system or time series therefrom) change with the scale at which the object is observed (Mandelbrot, 1983). A geometrically intuitive definition is then an exponent quantifying the scaling of an object bulk with its size as

5 bulk $\sim \operatorname{size}^{\mathrm{D}}$,

with bulk corresponding to a specific feature of the system (e.g., volume, area, mass, information content), and size corresponding to a linear scale (Theiler, 1990). As an example, the spatial volume (bulk) of a solid figure scales as the third-power of its length (size), hence $D=3$.

Given a discretely sampled realization (time series) from a $d$-dimensional dynamical system consisting of $N$ points in its phase-space and letting $\ell_{0}$ be the linear scale of the possibly underlying fractal structure, it is possible to define the following rescaling hierarchy:

1. on the coarsest scale $\ell_{0}$, the $N$ points are enclosed in a volume $V_{0}=\ell_{0}^{d}$;

2. on a finer scale $s_{\alpha}$, there are $M_{\alpha}$ boxes of a volume $V_{\alpha}=\left(\ell_{0} / s_{\alpha}\right)^{d}$ each of which contain $N_{\alpha}$ points.

In this way, we can cover the phase-space with boxes of different sizes $\ell_{\alpha}=\ell_{0} / s_{\alpha}$ and we can define a suitable (normalized) 15 measure in terms of the probability $p_{\alpha}$ for the system to fall in one of the boxes as $p_{\alpha}=N_{\alpha} / N$. Thus, all generalized fractal dimensions can be evaluated as the scaling exponent of the $q$-th order statistical moments of the probability density $p_{\alpha}$, i.e.,

$\sum_{\alpha} p_{\alpha}^{q} \sim \ell_{\alpha}^{D_{q}}$. 
An example of covering the phase-space with boxes of different size is shown in Fig. S1 for the 3-D projection of the full system attractor of the low order ocean-atmosphere model in the subspace $\left(T_{o, 2}, \Psi_{o, 2}, \psi_{a, 1}\right)$ for $C=0.015 \mathrm{~kg} \mathrm{~m}^{-2} \mathrm{~s}^{-1}$.
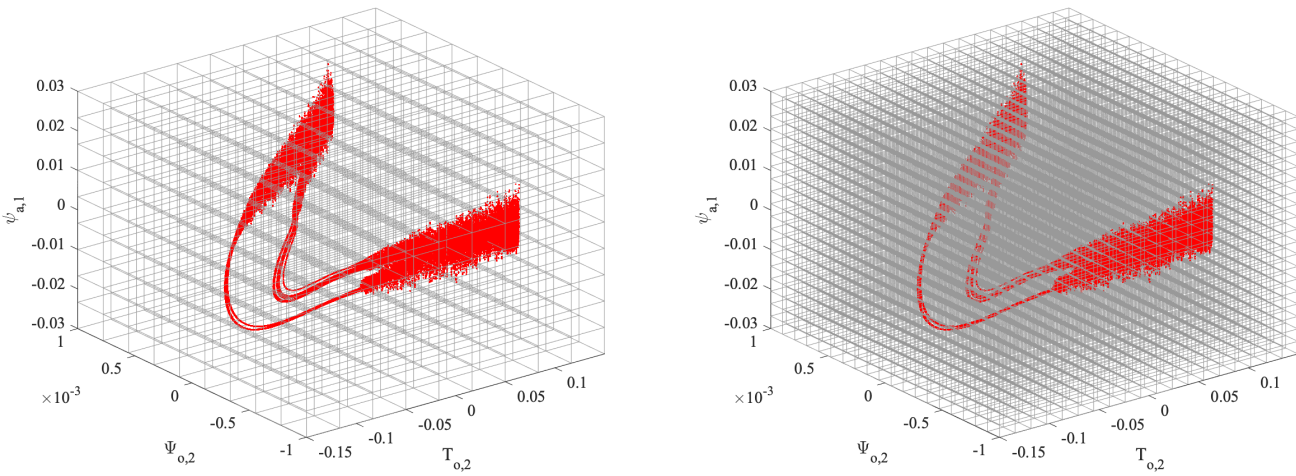

Figure S1. An example of covering the phase-space with boxes of different size: $s_{\alpha}=10$ (left) and $s_{\alpha}=20$ (right).

Based on partitioning the phase-space into cubes of different sizes $\ell_{\alpha}$, we can evaluate how $\sum_{\alpha} p_{\alpha}^{q}$ behaves with $\ell_{\alpha}$. As reported in Fig. S2 for $q \in[0,2]$ a scaling-law behavior is found whose exponent, in the limit $\ell_{\alpha} \rightarrow 0$, is the corresponding $q$-th order generalized fractal dimension $D_{q}$ (Grassberger, 1983; Hentschel and Procaccia, 1983).

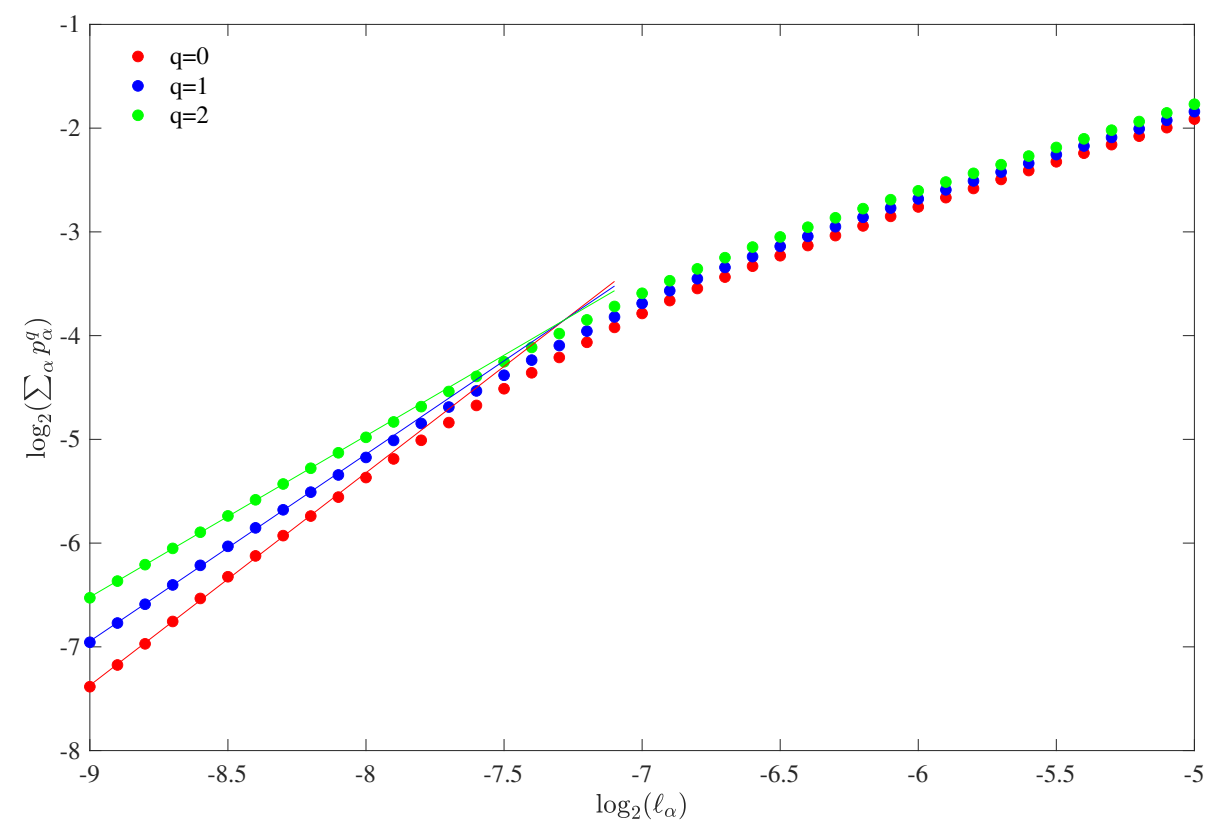

Figure S2. The log-log scaling plots of $\sum_{\alpha} p_{\alpha}^{q}$ for $q \in[0,2]$. The lines refer to the power law fit in the limit $\ell_{\alpha} \rightarrow 0$. 


\section{S2 Scaling plots at different scales}

As described in the main manuscript, our formalism allows us to investigate scale-dependent dynamical invariants and complexity measures by firstly identifying the intrinsic oscillations by using the Multivariate Empirical Mode Decomposition (MEMD) and then by investigating the phase-space properties at different scales by deriving the generalized dimensions. Thus, for each scale $\tau$ we can define

$F_{j^{\star}}(t)=\sum_{j=1}^{j^{\star}} \mathbf{c}_{j}(t)$

from which we can introduce the scale-dependent generalized dimensions $D_{q, \tau}$ as the scaling exponent of the $q-$ th order statistics of the phase-space coverage. As shown in Figs. S3-S4, a scaling-law behavior is found at different scales. As an illustrative example, we only show here the correlation integral, since it can commonly be more easily (and faster) evaluated than other moments (cfr. Grassberger, 1983).

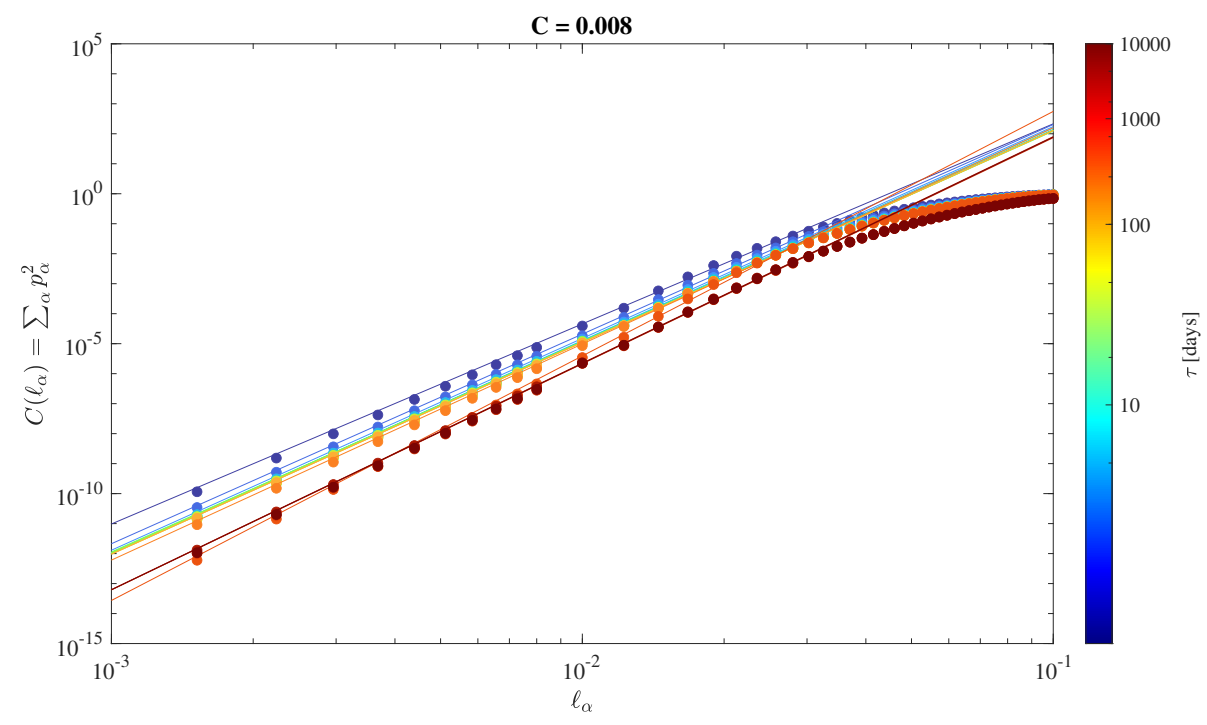

Figure S3. The log-log scaling plots of the correlation integral $C\left(\ell_{\alpha}\right)=\sum_{\alpha} p_{\alpha}^{2}$ as a function of the spatial scale $\ell_{\alpha}$ at different time scales as reported by colors for the case $C=0.008$. The lines refer to the power law fit in the limit $\ell_{\alpha} \rightarrow 0$.

For the considered low order ocean-atmosphere model, we find that there exists at least an order of magnitude in scale over which a scaling behavior is observed. A similar behavior is also observed when considering the reanalysis data as shown in Fig. S5 for the three different regions.

\section{S3 Divergence of higher-order moments}

When working with scale invariant features, an important point is to find the highest moment $q_{D}$ for which the estimated scaling law is statistically reliable, i.e., avoiding to retain spurious information due to the so-called "second order multifractal phase 


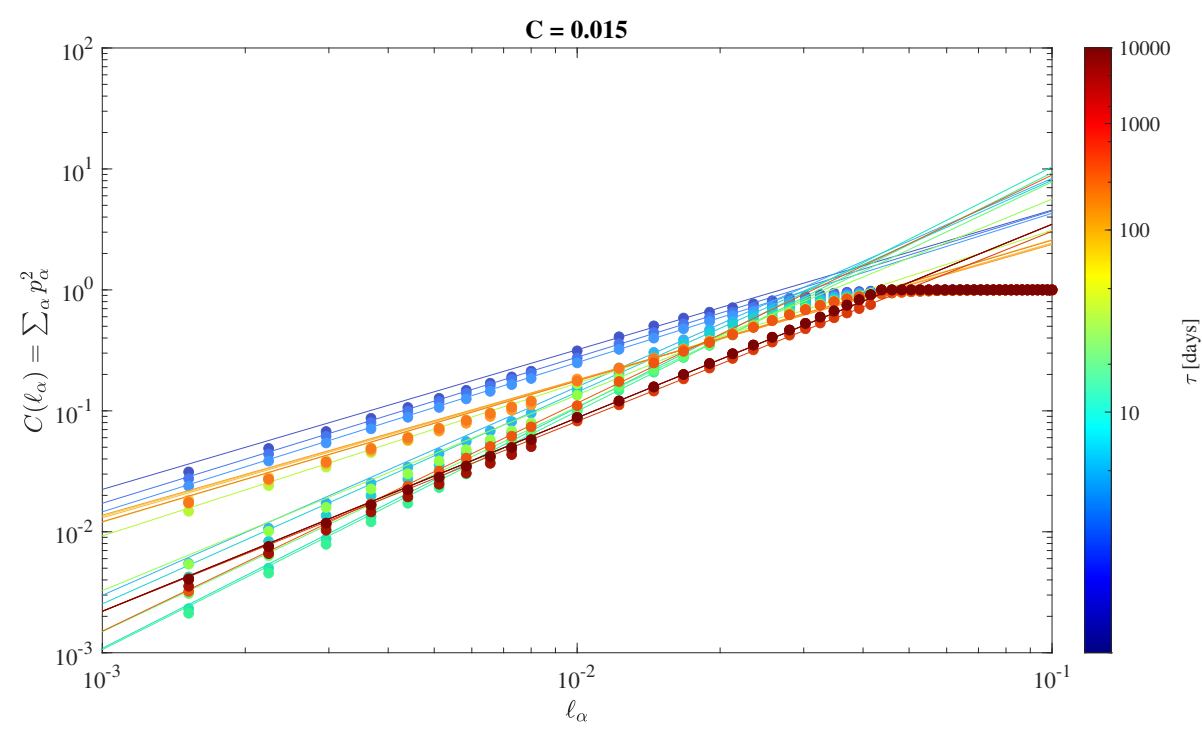

Figure S4. Same as in Fig. S3, but for $C=0.015$.
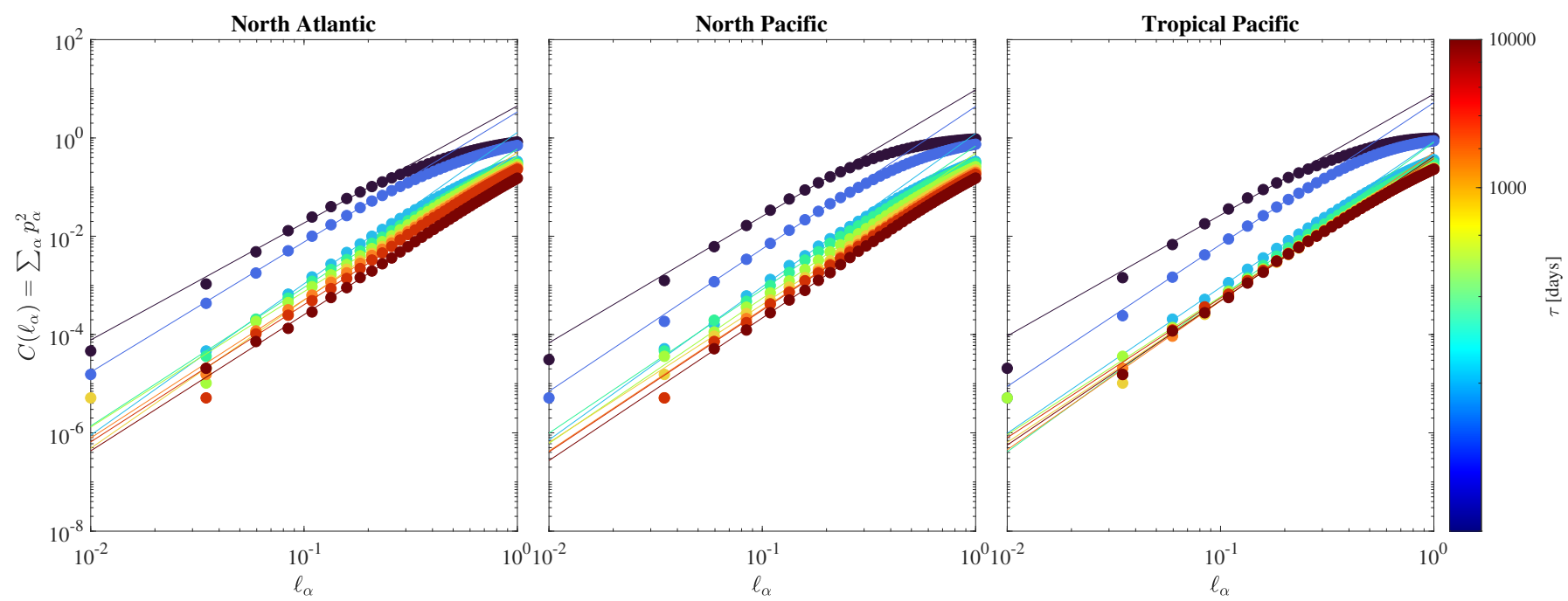

Figure S5. Same as in Fig. S3, but for the reanalysis data of the three considered ocean regions.

transition" or to the statistics of the very sparsely populated regions of phase space (see, e.g., Ch. 5 of Lovejoy and Schertzer, 2013). To address this problem and to support the statistical significance of our results, we have followed the approach also described in (Ch. 5 of Lovejoy and Schertzer, 2013) to evaluate the maximum moments as those derived from the tail of the cumulative distribution function of the data. Since we deal with the investigation of scale-dependent fractal dimensions, we evaluate the cumulative statistics at different scales. As shown in Figs. S6-S7, we observe that extreme fluctuations follow a power law decay leading to the divergence of the 6-th order moment and the 4-th order moment for $C=0.008$ and $C=0.015$, 
45 respectively (see Fig. S8). Thus, we fix our range of moments $-6<q<6$ and $-4<q<4$ for $C=0.008$ and $C=0.015$, respectively.

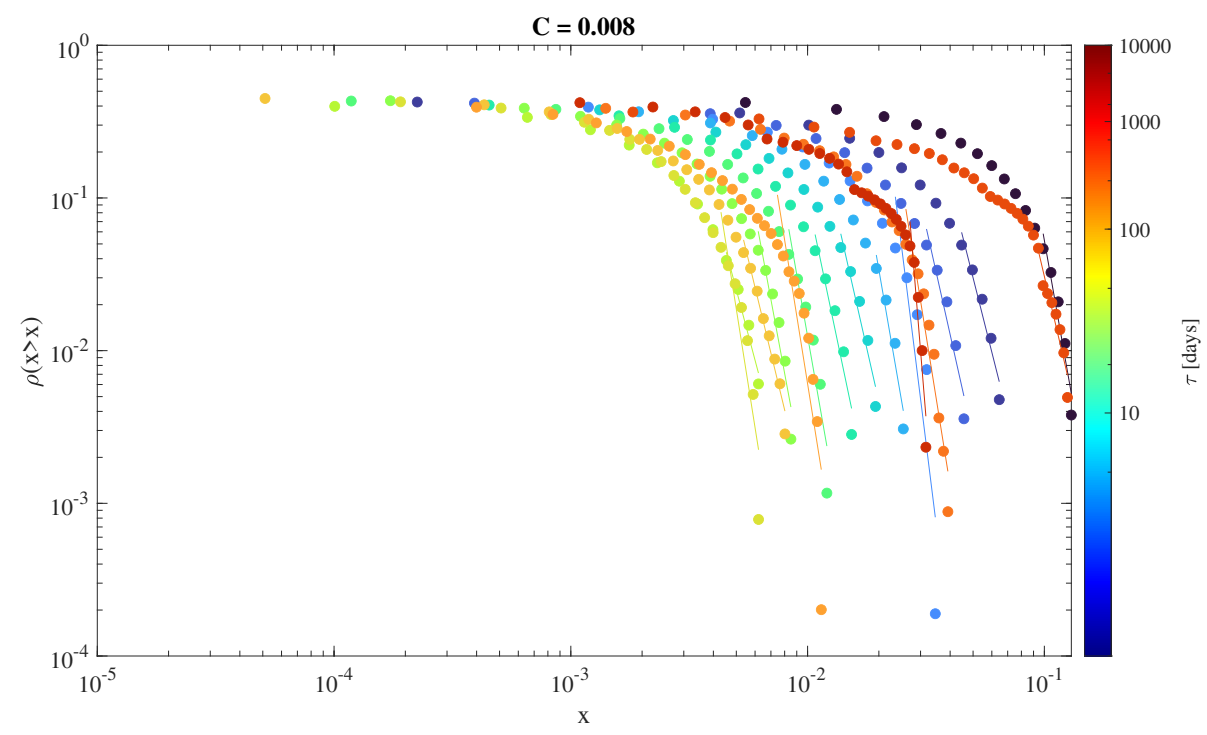

Figure S6. Complementary cumulative distribution functions at different scales as reported by different colors for the case $C=0.008$. The lines refer to the power law fit of the tail.

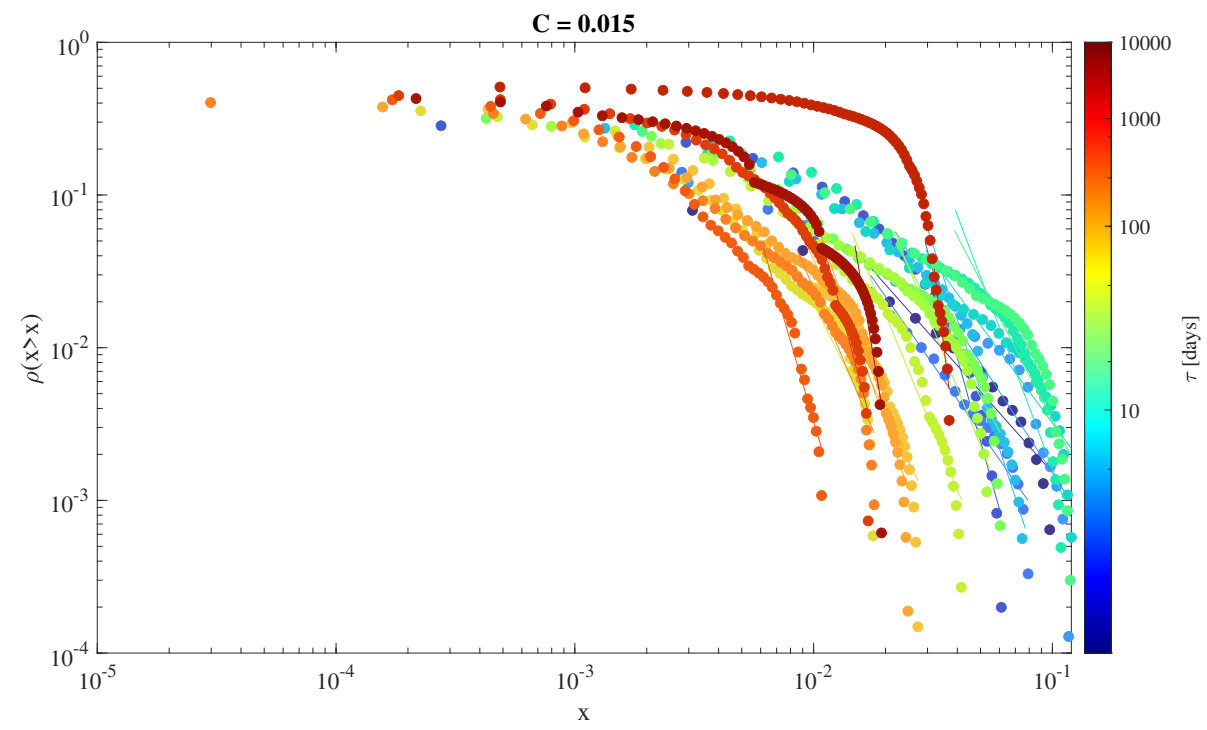

Figure S7. Same as in Fig. S6, but for $C=0.015$.

Similar results are also obtained for the reanalysis data (see Figs. S9-S10), for which we accordingly fix the range of considered moments to $-3<q<3$. 


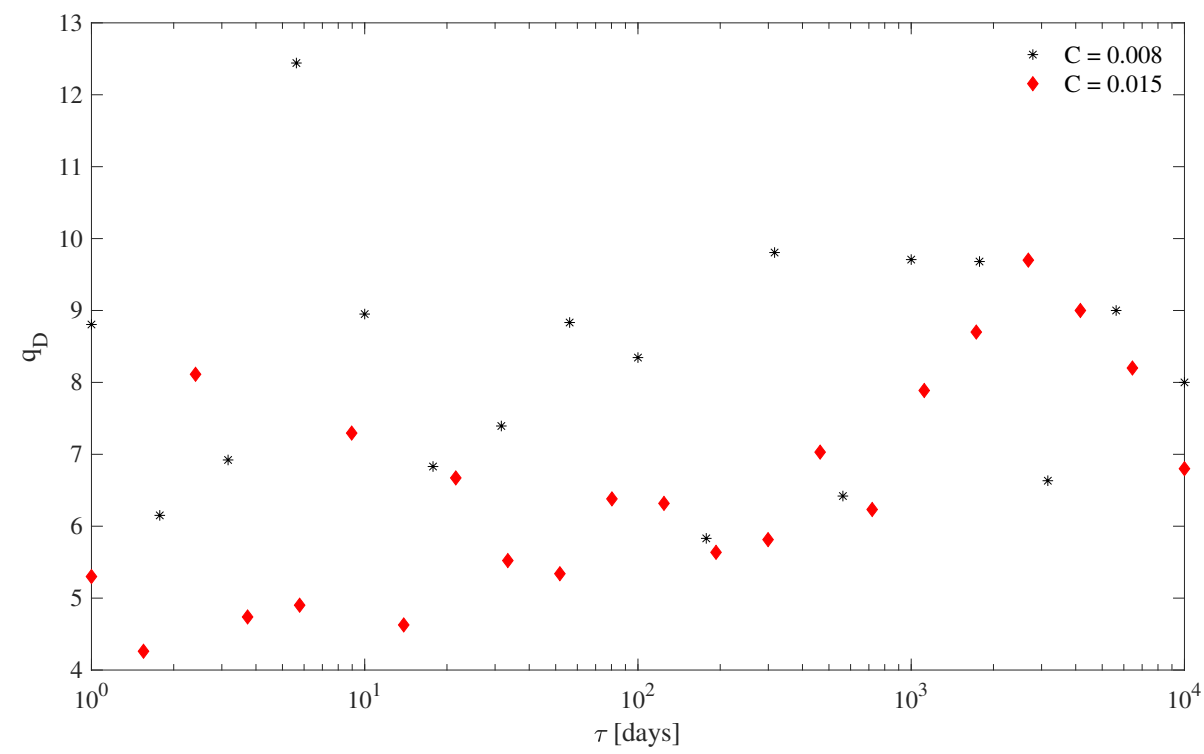

Figure S8. The power law scaling exponent $q_{D}$ as a function of the different scales for the case $C=0.008$ (black asterisks) and $C=0.015$ (red diamonds). The minimum $q_{D}$ has been chosen to set the range of statistically significant moments.

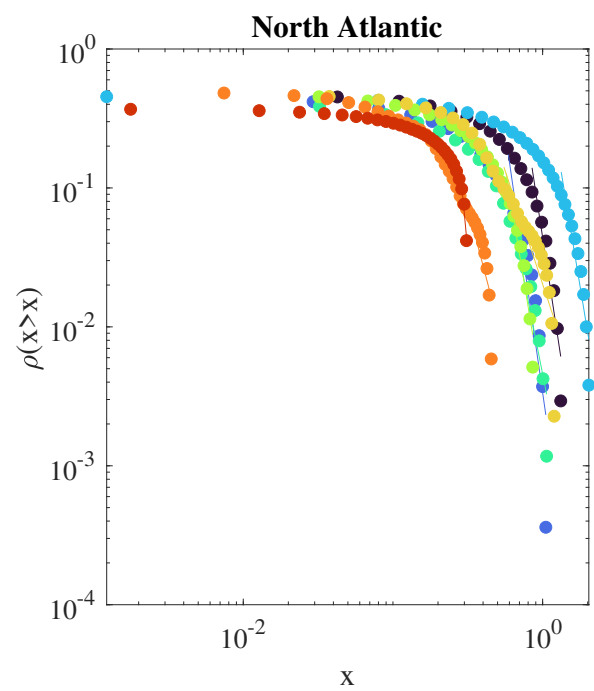

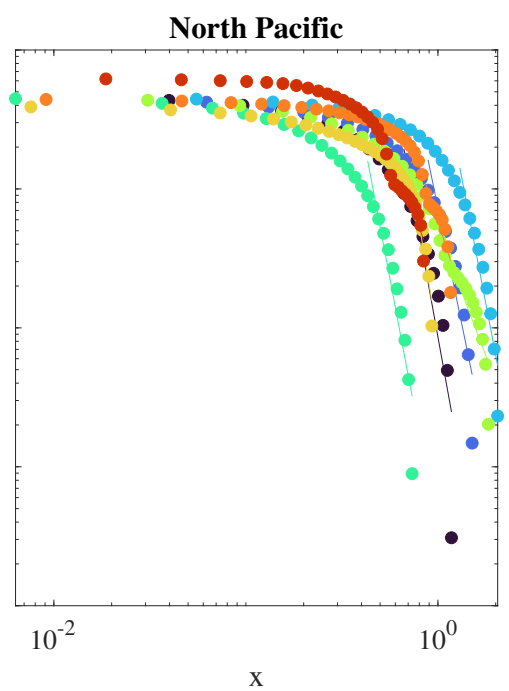

X

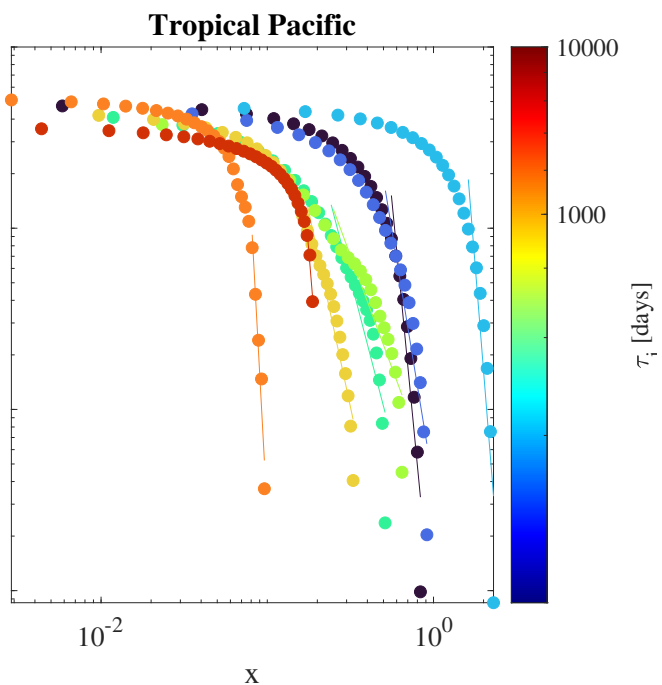

Figure S9. Same as in Fig. S6, but for the reanalysis data. 


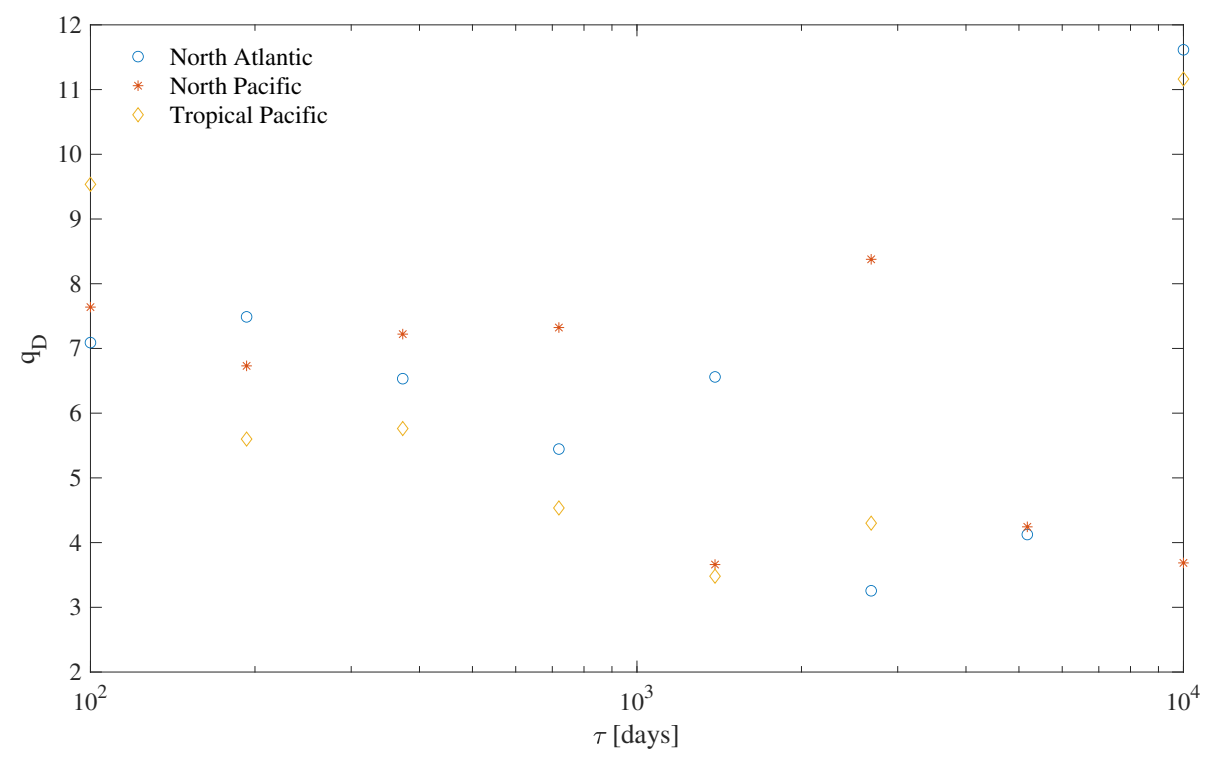

Figure S10. Same as in Fig. S8, but for the reanalysis data.

\section{S4 Lyapunov exponents}

50 Figures S11 reports the Lyapunov exponents separately for the ocean and the atmosphere components of the model for both values of the friction coefficient. We observe that for $C=0.008$ the instability of the atmosphere is large as compared the other value $C=0.015$. A Lyapunov dimension can be estimated as

$D_{L}=j+\frac{\sum_{i=1}^{j} \lambda_{i}}{\left|\lambda_{j+1}\right|}$

where $j$ is the number of positive and zero exponents, and $\lambda_{i}$ the $i$-th exponent. For the atmosphere, the Lyapunov dimension $D_{L} \sim 10$ for $C=0.008$, while for $C=0.015$ the atmospheric instability is weaker, and the Lyapunov dimension is slightly larger than 4. Note that for the ocean modes, the numerical estimates of all Lyapunov exponents are below zero, being more negative for the larger friction coefficient compatible with the corresponding behavior of the atmospheric modes. For the Lyapunov dimension, the two first exponents (although negative) are very close to 0 , and could be considered practically indistinguishable from zero. We therefore estimate the Lyapunov dimension of the ocean to 2. 

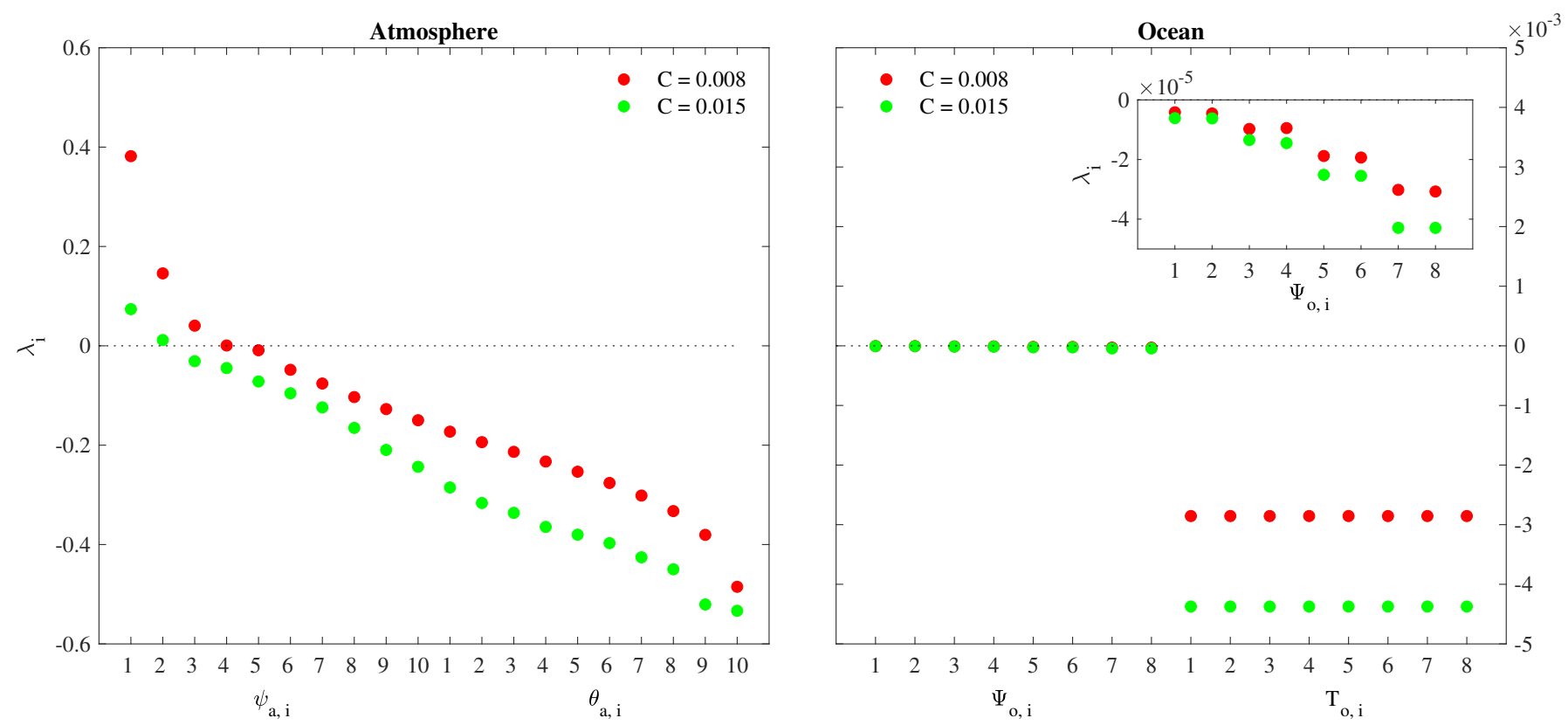

Figure S11. Lyapunov exponents $\lambda_{i}$ for the atmosphere (left) and the ocean (right) for $C=0.008$ (red) and $C=0.015$ (green). The inset is a zoom to better underline that the two first Lyapunov exponents are very close to zero.

\section{References}

Grassberger, P.: Generalized dimensions of strange attractors, Phys. Lett. A, 97, 227-230, 1983.

Hentschel, H. G. E. and Procaccia, I.: The infinite number of generalized dimensions of fractals and strange attractors, Physica D, 8, 435-444, 1983.

Lovejoy, S. and Schertzer, D.: The Weather and Climate: Emergent Laws and Multifractal Cascades, 496 pp., Cambridge University Press, 2013.

Mandelbrot, B. B.: The fractal geometry of nature, W.H. Freeman and Company, 468 pp., ISBN 9780716711865, 1983.

Theiler, J.: Estimating fractal dimension, J. Opt. Soc. Am. A, 7, 1055-1073, 1990. 